\title{
Effect of the Coulomb interaction on the electron relaxation of weakly-confined quantum dot systems using the full configuration interaction approach
}

\author{
Juan I. Climente, ${ }^{1, *}$ Andrea Bertoni, ${ }^{1}$ Massimo Rontani, ${ }^{1}$ \\ Guido Goldoni, ${ }^{1,2}$ and Elisa Molinari ${ }^{1,2}$ \\ ${ }^{1}$ CNR-INFM S3, Via Campi 213/A, 41100 Modena, Italy \\ ${ }^{2}$ Dipartamento di Fisica, Università degli Studi di Modena e Reggio Emilia, Via Campi 213/A, 41100 Modena, Italy
}

(Received 10 May 2006; revised manuscript received 20 June 2006; published 7 September 2006)

\begin{abstract}
We study acoustic-phonon-induced relaxation of charge excitations in single and tunnel-coupled quantum dots containing few confined interacting electrons. The full configuration interaction approach is used to account for the electron-electron repulsion. Electron-phonon interaction is accounted for through both deformation potential and piezoelectric field mechanisms. We show that electronic correlations generally reduce intradot and interdot transition rates with respect to corresponding single-electron transitions, but this effect is lessened by external magnetic fields. On the other hand, piezoelectric field scattering is found to become the dominant relaxation mechanism as the number of confined electrons increases. Previous proposals to strongly suppress electron-phonon coupling in properly designed single-electron quantum dots are shown to hold also in multielectron devices. Our results indicate that few-electron orbital degrees of freedom are more stable than single-electron ones.
\end{abstract}

DOI: 10.1103/PhysRevB.74.125303

PACS number(s): 73.21.La, 73.61.Ey, 72.10.Di, 73.22.Lp

\section{INTRODUCTION}

Recent experiments have appointed coupling to acoustic phonons as the main source of electron relaxation in the excited states of semiconductor quantum dots (QDs) with weak lateral confinement ${ }^{1,2}$ and coupled quantum dots (CQDs) with small tunneling energy, ${ }^{3-5}$ i.e., with the electronic relevant energy scale in the few-meV range. This usually restricts lifetimes to the order of nanoseconds, ${ }^{1}$ imposing severe limitations on the performance of QD devices which rely on the coherent dynamics of electron charge states. A prominent example are charge qubits, whose main disadvantage, as compared to spin qubits, is the faster decoherence rate. ${ }^{1}$

As a first step to eventually gain control over charge relaxation rates, several theoretical works have investigated the fundamental physics of electron-phonon coupling in QD structures. Bockelmann described the qualitative effect of lateral (spatial and magnetic) confinement on the electron transition rates in QDs. ${ }^{6}$ The domains of deformation potential (DP) and piezoelectric field (PZ) interactions, the two main scattering mechanisms leading to interaction with acoustic phonons, were established for both QD and CQD structures. ${ }^{7-10}$ Building on these works, methods to minimize electron-phonon coupling in QDs and vertically CQDs were recently proposed which may bring electron lifetimes in the range of microseconds, thus making them comparable to usual spin relaxation rates. ${ }^{7,11-13}$

Noteworthy, all of the aforementioned experimental and theoretical works studied charge relaxation at a singleelectron (SE) level. So far, multielectron (ME) systems have been essentially restricted to the context of spin relaxation, , 2,14,15 and only lately charge relaxation processes in ME systems started to be considered. ${ }^{16,17}$ In particular, in Ref. 17 we have investigated Coulomb correlated systems, and we have reported numerical evidence that electronic correlations generally lead to reduced decay rates of the excited electronic states in weakly confined dots, as compared to independent-particle estimates. This finding suggests that ME devices might better exploit the discrete energy spectrum of QDs.

In this paper, we extend our previous investigation (Ref. 17) analyzing in detail the mechanisms by which Coulomb interaction affects charge relaxation rates in ME QD systems. We also investigate the effect of external magnetic fields on intradot transition rates, and isospin transition rates in $\mathrm{ME}$ vertically CQDs. Electron-phonon interaction is included through both DP and PZ scattering channels (the latter was neglected in Ref. 17 as well as in many theoretical investigations).

The paper is organized as follows. In Sec. II we describe our theoretical model. In Sec. III we study ME charge relaxation in single QDs as a function of the lateral (spatial and magnetic) confinement, and interpret the correlation-induced scattering reduction in terms of the SE configuration mixing. The applicability in ME QDs of magnetic-field-based suggestions for controlling charge relaxation rates, previously reported for SE structures, 7,12,13 is addressed in this section. In Sec. IV we investigate the effect of interdot distance on the isospin transition rate of ME vertically CQDs, and finally in Sec. V we summarize our results.

\section{THEORETICAL FORMULATION}

The theoretical model we use is similar to that described in Ref. 7 for single-electron excitations, but now considering $N$-electron states $(N=1-5)$. We study disk-shaped QDs where the lateral confinement is much weaker than the vertical one, and the dot and surrounding barrier are made of materials with similar elastic properties. Several QD structures reported in the literature fit this description. ${ }^{1,2,18}$

A number of useful approximations can be made for such QDs. First, since the weak lateral confinement gives interlevel spacings within the range of a few meV, only acoustic 
phonons have significant interaction with bound carriers, while optical phonons can be safely neglected. Second, the elastically homogeneous materials are not expected to induce phonon confinement, which allow us to consider only bulk phonons. Finally, the different energy scales of vertical and lateral electronic confinement allow us to decouple vertical and lateral motion in the building of SE spin-orbitals. We then take a parabolic confinement profile in the in-plane $(x, y)$ direction, with single-particle energy gaps $\hbar \omega_{0}$, which yields the Fock-Darwin states. ${ }^{19}$ In the vertical direction $(z)$ the confinement is provided by a rectangular quantum well of length $L_{z}$, with the barrier height determined by the bandoffset between the QD and barrier materials. The quantum well solutions are derived numerically. Spin-orbit coupling is neglected in this work, since the long spin relaxation times measured in QD structures similar to those we study here indicate that the spin degrees of freedom are well-separated from the orbital ones. ${ }^{1}$ Therefore the SE spin-orbitals can be written as

$$
\psi_{\alpha}(x, y, z ; \sigma)=\phi_{n, m}(x, y) \xi_{g}(z) \chi_{\sigma},
$$

where $\phi_{n, m}$ is the $n$th Fock-Darwin orbital with azimuthal angular momentum $m, \xi_{g}$ is the symmetric quantum well solution with parity $g$ with respect to the reflection about the $z=0$ plane, with $g=0(1)$ denoting even (odd) parity, and $\chi_{\sigma}$ is the spinor eigenvector of the spin $z$-component with eigenvalue $\sigma(\sigma= \pm 1 / 2)$. We will also label Fock-Darwin states with the standard notation $n l$, where $l=s, p_{ \pm}, d_{ \pm}, \ldots$ correspond to $m=0, \pm 1, \pm 2, \ldots$, respectively.

As for the inclusion of Coulomb interactions, we need to go beyond the Hartree-Fock approximation in order to include electronic correlations, which are critical in phononinduced electron scattering processes. ${ }^{16}$ Moreover, since we are interested in the relaxation time of excited states, we need to know both ground and excited states with comparable accuracy. Our method of choice is the full configuration interaction approach: the ME wave functions are written as linear combinations $\left|\Psi_{a}\right\rangle=\Sigma_{i} c_{a i}\left|\Phi_{i}\right\rangle$, where the Slater determinants $\left|\Phi_{i}\right\rangle=\Pi_{\alpha_{i}} c_{\alpha_{i}}^{\dagger}|0\rangle$ are obtained by filling in the SE spin-orbitals $\alpha$ with the $N$ electrons in all possible ways consistent with symmetry requirements; here $c_{\alpha}^{\dagger}$ creates an electron in the level $\alpha$. The ME ground and excited states can then be labeled by the total angular momentum $z$ component $M=0, \pm 1, \pm 2, \ldots$, total parity $G=0,1$, total spin $S$, and total spin $z$-projection $S_{z}$. The fully interacting Hamiltonian is numerically diagonalized, exploiting orbital and spin symmetries. $^{20}$

We assume zero temperature, which suffices to capture the main features of one-phonon processes. ${ }^{12}$ At this temperature, only phonon emission processes are present. We evaluate the relaxation rate between selected initial (occupied) and final (unoccupied) ME states, $b$ and $a$, using the Fermi golden rule:

$$
\tau_{b \rightarrow a}^{-1}=\frac{2 \pi}{\hbar} \sum_{\nu \mathbf{q}}\left|\sum_{i j} c_{b i}^{*} c_{a j}\left\langle\Phi_{i}\left|V_{\nu \mathbf{q}}\right| \Phi_{j}\right\rangle\right|^{2} \delta\left(E_{b}-E_{a}-E_{q}\right),
$$

where the electron states $\left|\Psi_{\beta}\right\rangle(\beta=a, b)$ have been written explicitly as linear combinations of Slater determinants, $V_{\nu \mathbf{q}}$ is the interaction operator of an electron with an acoustic phonon of momentum q via deformation potential $(\nu=D P)$ or piezoelectric field ( $\nu=P Z$ ) interaction, $E_{\beta}$ stands for the $\beta$ electron state energy and $E_{q}$ represents the phonon energy.

The electron-phonon interaction matrix element can be written more explicitly as

$$
\left\langle\Phi_{i}\left|V_{\nu \mathbf{q}}\right| \Phi_{j}\right\rangle=M_{\nu}(\mathbf{q})\left\langle\Phi_{i}\left|e^{-i \mathbf{q r}}\right| \Phi_{j}\right\rangle,
$$

where the rightmost term is the electron form factor and $M_{\nu}(\mathbf{q})$ is a prefactor which depends on the scattering mechanism $\nu$. For a zinc-blende crystal the prefactors are given by: ${ }^{7}$

(i) the electron-longitudinal acoustic (LA) phonon scattering due to the deformation potential ( $\nu=\mathrm{LA}-\mathrm{DP})$ :

$$
\left|M_{\mathrm{LA}-\mathrm{DP}}(\mathbf{q})\right|^{2}=\frac{\hbar D^{2}}{2 d c_{\mathrm{LA}} \Omega}|\mathbf{q}|,
$$

(ii) the electron-LA phonon scattering due to the PZ field $(\nu=\mathrm{LA}-\mathrm{PZ})$ :

$$
\left|M_{\mathrm{LA}-\mathrm{PZ}}(\mathbf{q})\right|^{2}=\frac{32 \pi^{2} \hbar e^{2} h_{14}^{2}}{\varepsilon^{2} d c_{\mathrm{LA}} \Omega} \frac{\left(3 q_{x} q_{y} q_{z}\right)^{2}}{|\mathbf{q}|^{7}},
$$

(iii) the electron-transversal acoustic (TA) phonon scattering due to the PZ field ( $\nu=\mathrm{TA}-\mathrm{PZ})$ :

$$
\begin{aligned}
\left|M_{\mathrm{TA}-\mathrm{PZ}}(\mathbf{q})\right|^{2}= & \frac{32 \pi^{2} \hbar e^{2} h_{14}^{2}}{\varepsilon^{2} d c_{\mathrm{TA}} \Omega} \\
& \times\left|\frac{q_{x}^{2} q_{y}^{2}+q_{y}^{2} q_{z}^{2}+q_{z}^{2} q_{x}^{2}}{|\mathbf{q}|^{5}}-\frac{\left(3 q_{x} q_{y} q_{z}\right)^{2}}{|\mathbf{q}|^{7}}\right| .
\end{aligned}
$$

In the above expressions $D, d$, and $\Omega$ stand for the crystal acoustic deformation potential constant, density, and volume, respectively. $e$ is the electron charge, $h_{14}$ the PZ constant, and $\varepsilon$ the static dielectric constant. The sum in Eq. (2) runs twice over $\nu=$ TA-PZ, to account for the two transverse phonon modes. It is worth noting that, whereas for DP scattering $M_{D P} \propto \sqrt{|\mathbf{q}|}$, for PZ scattering $M_{P Z} \propto 1 / \sqrt{|\mathbf{q}|}$. As a result, DP scattering is dominant when the emitted phonon energy is sufficiently large, while PZ scattering dominates at small phonon energy. For SE transitions in GaAs QDs, even in the weakly-confined regime, the DP mechanism usually prevails in the absence of external fields. However, we have recently shown ${ }^{7}$ that the PZ mechanism may rapidly become dominant in the presence of a vertical magnetic field which tends to suppress the single-particle gaps. Another qualitative difference between DP and PZ mechanisms is that the latter depends explicitely on the direction of propagation of the phonon through the phonon momentum components $q_{x}, q_{y}$, $q_{z}$, whereas the former depends only on the modulus $|\mathbf{q}|$. This may lead to significantly different behaviors under certain circumstances, as we shall see below.

One can see from the above expressions that Coulomb interaction influences electron scattering with phonons in two ways. First, it introduces changes in the electron energy gaps $E_{b}-E_{a}$, and hence in the energy and momentum of the emitted phonon. ${ }^{16}$ Second, it introduces changes in the orbital part of the electron state, hence changing the electronphonon wave functions coupling. The latter effect is reflected 
in Eq. (2) through the Slater determinant coefficients. Since these coefficients strongly depend on the number of particles, the regime of correlation, and the presence of external fields, important changes in the ME relaxation rates should be expected when varying these parameters.

In this work we consider mostly relaxation rates corresponding to the fundamental spin-conserving transition in single and coupled QDs, i.e., transitions involving the ground state and the first excited state with the same $\left(S, S_{z}\right)$ quantum numbers. This transition could be monitored, e.g., by means of pump-and-probe techniques, ${ }^{1,2,14}$ since relaxation to or from any intermediate state with different spin should be much slower and therefore it will barely interfere.

Below we shall investigate $\mathrm{GaAs} / \mathrm{Al}_{0.3} \mathrm{Ga}_{0.7} \mathrm{As}$ QDs, using the following material parameters: ${ }^{21}$ electron effective mass $m^{*}=0.067$, band-offset $V_{c}=243 \mathrm{meV}$, crystal density $d=5310 \mathrm{~kg} / \mathrm{m}^{3}$, acoustic deformation potential constant $D$ $=8.6 \mathrm{eV}$, effective dielectric constant $\varepsilon=12.9$, and piezoelectric constant $h_{14}=1.41 \times 10^{9} \mathrm{~V} / \mathrm{m}$. For the sound speed $c_{\sigma}$, we take into account that generally in cylindrical QDs most of the scattering arises from phonon propagation close to the growth direction. ${ }^{13}$ We then assume that the QDs are grown along the $\left[\begin{array}{lll}1 & 0 & 0\end{array}\right]$ direction and use the corresponding values $c_{\mathrm{LA}}=4.72 \times 10^{3} \mathrm{~m} / \mathrm{s}$ and $c_{\mathrm{TA}}=3.34 \times 10^{3} \mathrm{~m} / \mathrm{s} .{ }^{22}$ In our calculations we deal with QDs with lateral confinement energies which in some cases are rather weak $\left(\hbar \omega_{0}<1 \mathrm{meV}\right)$. Wellconverged few-body states are obtained for such structures using a basis set composed by the Slater determinants which result from all possible combinations of $62 \mathrm{SE}$ spin-orbitals with $N$ electrons. Due to the strong confinement in the vertical direction, only the lowest $g=0$ (for single QDs) or the lowest $g=0,1$ (for CQDs) eigenstates are included in the single-particle basis.

\section{SINGLE QUANTUM DOTS AND MAGNETIC FIELD}

In this section we first study charge relaxation rates in QDs with $N$ interacting electrons as a function of the harmonic lateral confinement originated by electrostatic fields, and next consider the effect of adding a magnetic field. To study the lateral confinement, we vary the characteristic frequency of the confining parabola in the $\hbar \omega_{0} \sim 0-6 \mathrm{meV}$ range, thus moving from a strongly to a rather weakly correlated regime. Figure 1 depicts the corresponding results for $N=1-5$ electrons in QDs with height $L_{z}=10 \mathrm{~nm}$. It can be observed that for all $N$ the qualitative shape of the relaxation rate curve is similar to that of the SE case (top panel): it shows two maxima, connected with the PZ and DP scattering mechanisms, and it vanishes at small and large confinement energies due to the small phonon density and small electronphonon coupling, respectively. ${ }^{6,7,23}$ Another trend observed in Fig. 1 is the shift of the scattering rate maxima towards larger confinement energies with increasing number of electrons, as well as the increasing relative height of the $\mathrm{PZ}$ maximum. Both features follow from the increasing density of states with larger $N$, which leads to smaller interlevel spacing and, therefore, to larger $\hbar \omega_{0}$ values associated with a given phonon energy. We illustrate this in Fig. 1 with downward arrows to point at the confinement energies which give

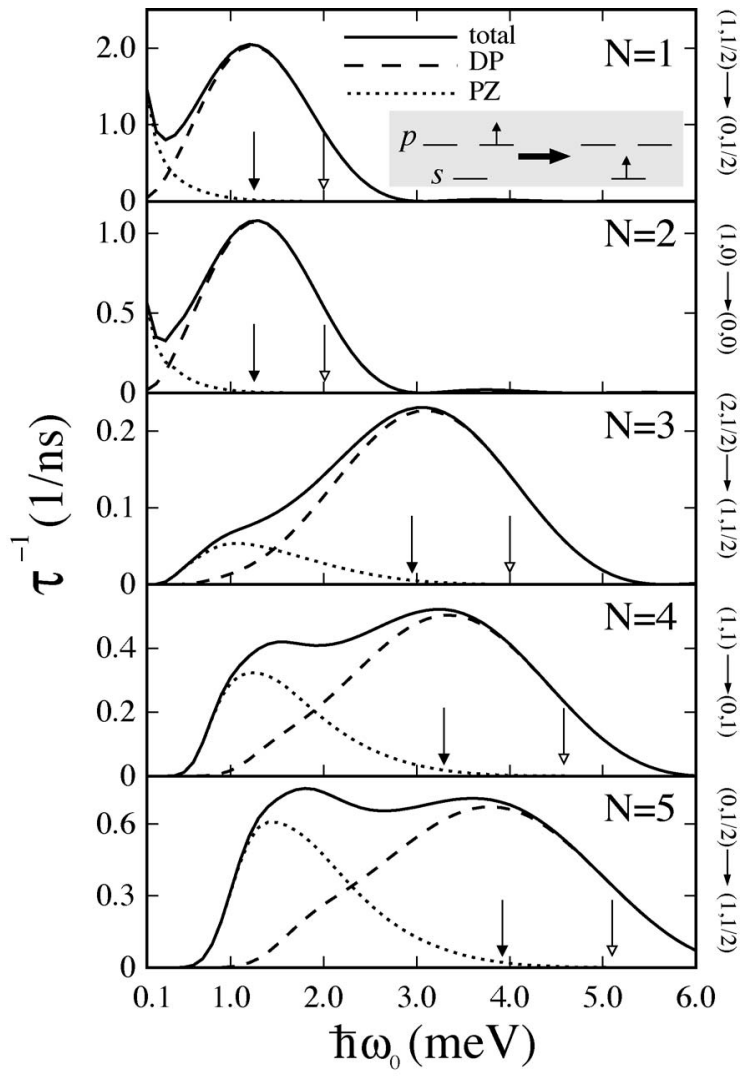

FIG. 1. Charge relaxation rate vs lateral confinement for a QD with vertical width $L_{z}=10 \mathrm{~nm}$ filled with $N=1-5$ electrons. Solid lines: total scattering rate. Dashed line: DP contribution. Dotted lines: PZ contribution. Note the different vertical scale in each panel. Next to the right axis, we indicate the quantum numbers $(M, S)$ of the states involved in the transition. The downward arrows in each panel point at the confinement energy leading to emitted phonon energies of 1.3 (solid arrowheads) and $2 \mathrm{meV}$ (empty arrowheads). Inset in the top panel: SE configurations involved in the $N=1$ transition.

two selected phonon energies, $E_{q}=1.3$ (solid arrowhead) and $E_{q}=2.0 \mathrm{meV}$ (empty arrowhead). It can be seen that there is a shift towards larger $\hbar \omega_{0}$ values with increasing $N .{ }^{24} \mathrm{An}$ important implication of this result is that $\mathrm{PZ}$ scattering, which is negligible except at very weak confinement energies in the SE picture, may actually become the dominant scattering mechanism for usual QD confinement energies in the ME case (see, e.g., $\hbar \omega_{0}=2 \mathrm{meV}$ in the $N=5$ picture). In light of this, some of the estimates in previous investigations, which studied electron-phonon coupling in weakly-confined QDs considering DP interaction only, may need a revision. ${ }^{16,17}$

One also observes in Fig. 1 that the phonon energy which gives maximum scattering is approximately constant, regardless of the number of electrons and lateral confinement. For example, $E_{q} \approx 1.3 \mathrm{meV}$ for the DP maximum. This is consistent with the fact that the preferential phonon emission in intradot transitions takes place around the vertical direction: 6,13 indeed, at $E_{q} \approx 1.3 \mathrm{meV}$ the longitudinal acoustic phonon wavelength gives maximum coupling with the electron wave function in the quantum well, $\xi_{g}$, which does 


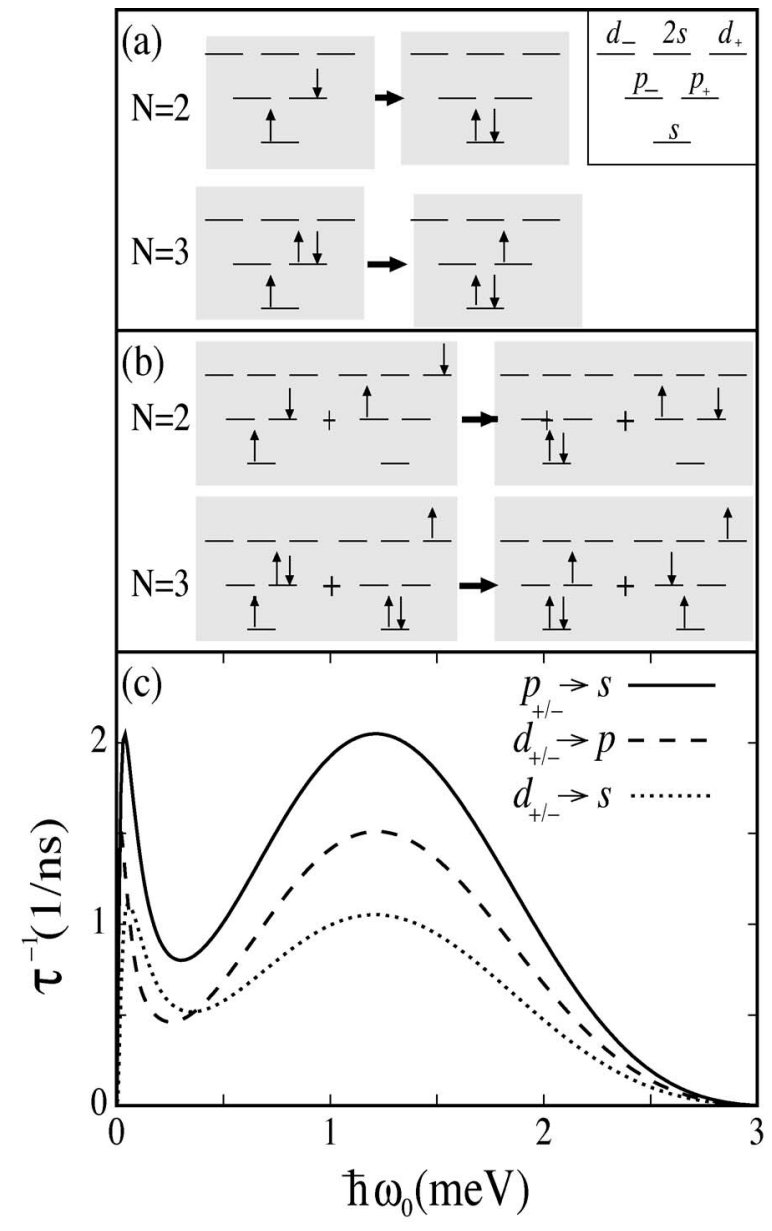

FIG. 2. SE electronic configurations (Slater determinants) involved in the fundamental transition for the QDs of Fig. 1 for $N$ $=2$ and 3 for (a) noninteracting and (b) interacting electrons. In the interacting case only the two most weighted configurations are shown. (c) SE scattering rates vs lateral confinement energy between selected Fock-Darwin orbitals at fixed phonon energy $E_{q}$ $=\hbar \omega_{0}$.

not depend on $\hbar \omega_{0}$ and is weakly affected by Coulomb interactions.

Figure 1 shows that the excited state lifetimes depend strongly on the number of carriers (note the different vertical scale of each panel), the shorter lifetimes being shown by the $N=1$ case. As a matter of fact, the $N=1$ transition, which corresponds to the $p \rightarrow s$ relaxation, represents the independent-particle limit of the ME cases shown in the same figure. For example, for $N=2$ an independent particle filling gives a $(M=0, S=0)$ ground state, with the two electrons occupying the $s$ spin-orbitals, and a $(M=1, S=0)$ excited state, with one electron in the $s$ orbital and another in the $p$ orbital [see electronic configuration diagrams in Fig. $2(\mathrm{a})]$. Thus the transition involves a one-electron scattering $p \rightarrow s$ orbital, while the other electron remains as a spectator. Similar reasonings apply to $N=3-5$. Therefore Fig. 1 shows that the Coulomb-interaction-free relaxation rate $(N=1$ panel) generally gives an upper bound to the actual rate when electron-electron interaction is taken into account. This result holds for all number of particles studied and most

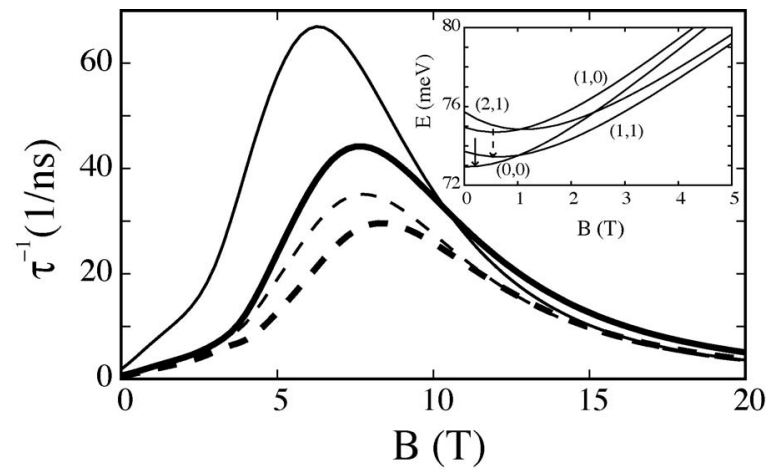

FIG. 3. Charge relaxation rate vs magnetic field for the lowest singlet (solid lines) and triplet (dashed lines) transitions for $N=2$ in a QD with $L_{z}=10 \mathrm{~nm}$ and $\hbar \omega_{0}=2 \mathrm{meV}$. Thick lines: interacting case. Thin lines: noninteracting case. Inset: lowest-lying energy levels and their quantum numbers $(M, S)$; arrows indicate the transitions under study.

confinement strengths, although the trend is nonmonotonic with $N$.

The reduction of the relaxation rate noted above can be explained in terms of SE configurations mixing. In order to illustrate this, we analyze in detail the $N=2$ and $N=3$ cases in Fig. 2, where panel (a) represents the electronic configurations of the first excited and ground states which follow from an independent-particle filling, while panel (b) represents the two most important configurations contributing to the same states when Coulomb interaction is included. By comparison, one can see that in the noninteracting picture only the $p \rightarrow s$ transition takes place, whereas in the interacting picture it is partially replaced by the $d \rightarrow s$ and $d \rightarrow p$ transitions. We then compare the relaxation rates of the individual SE scattering processes [panel (c)], fixing the transition energy $E_{q}=\hbar \omega_{0}$ in all cases in order to ensure that the comparison considers orbital effects only. One can see that $p \rightarrow s$ is the fastest transition. Therefore when it is partially replaced by $d \rightarrow s$ and $d \rightarrow p$ the overall relaxation rate is reduced. Obviously, the stronger the mixing of configurations the larger the reduction. This explains why the $N=3$ scattering rate is well below that of $N=2$ : the ground state electrons already occupy the $p$-shell and the kinetic energy difference with respect to the excited states is then much smaller, which allows stronger Coulomb-induced mixing. Analogous logic can be used to explain the scattering reduction in the $N>3$ systems.

We next investigate the effect of a magnetic field $B$, applied along the vertical direction of the QD, on the ME relaxation rate. The magnetic field is expected to introduce new physics because it strongly modifies the SE energy levels, which now draw the well-known Fock-Darwin spectrum. ${ }^{19}$ In particular, the states involved in the fundamental transition converge to the same (lowest) Landau level. This has important implications on the energy of the emitted phonon, which is reduced, the regime of electronic correlations, which become stronger, and the SE configurations of the low-lying ME states, which differ from those at zero magnetic field. In Fig. 3 we plot the relaxation rate corresponding to $N=2$ in a QD with lateral confinement 


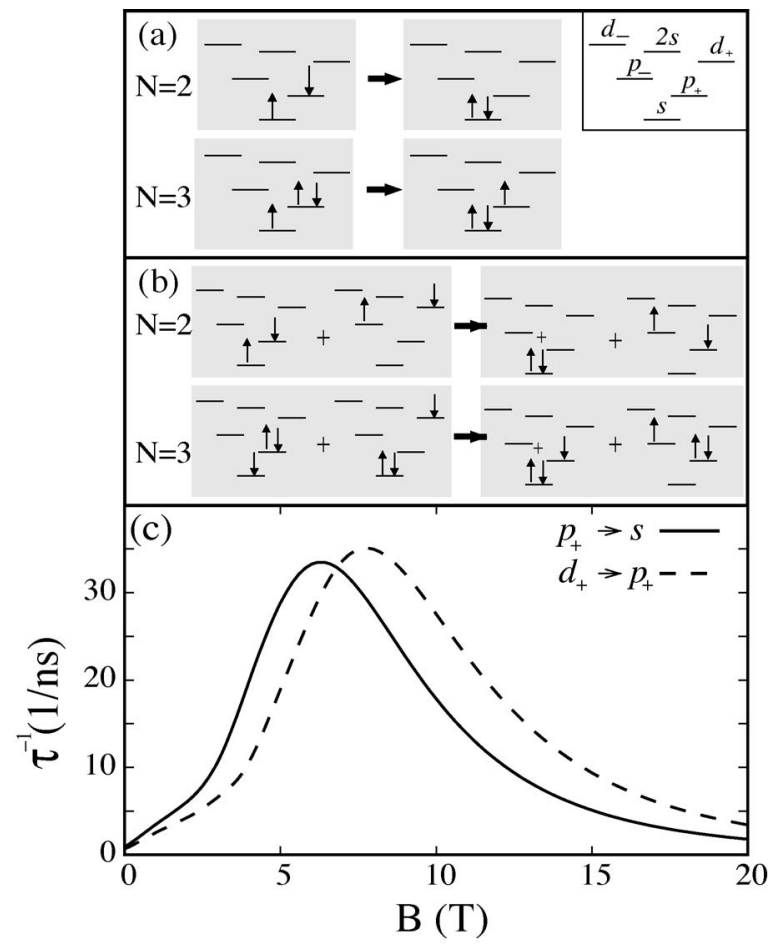

FIG. 4. SE electronic configurations (Slater determinants) involved in the fundamental transition for $N=2$ and 3 for (a) noninteracting and (b) interacting electrons in a magnetic field. In the interacting case only the two most weighted configurations are shown. (c) SE scattering rates between selected Fock-Darwin orbitals vs magnetic field. QD parameters are the same as in Fig. 3.

$\hbar \omega_{0}=2 \mathrm{meV}$ and width $L_{z}=10 \mathrm{~nm}$. We show the fundamental transition both in the singlet $(S=0)$ sector (solid lines) and in the triplet $(S=1)$ sector. We also compare the interacting and noninteracting case (thick and thin lines, respectively).

It can be seen in the figure that the shape of the ME curves is again qualitatively similar to that of the SE ones. From previous investigations, we know that the scattering rate at fields exceeding a few Tesla is largely determined by the PZ scattering channel. ${ }^{7}$ In addition, we note that the correlation-induced reduction of the relaxation rates changes with the field. Indeed, the factor of reduction tends to decrease as $B$ increases, and at some point $(B \approx 10.5 \mathrm{~T}$ for the singlet sector, $B \approx 13 \mathrm{~T}$ for the triplet sector) the effect of correlations is reversed, so that a small enhancement is found instead of the more common reduction. This reversal in the behavior can be explained in terms of the mixing of configurations in the presence of a magnetic field. When no Coulomb interaction is considered only the $p_{+} \rightarrow s$ SE transition contributes to the total scattering, as shown in Fig. 4(a). However, if we take into account the Coulomb-induced mixing between the two most important configurations, Fig. 4(b), new relaxation channels are opened, notably the $d_{+}$ $\rightarrow s$ and $d_{+} \rightarrow p_{+}$SE transitions. The increasing relative weight of these transitions with $B$, along with the fact that the $d_{+} \rightarrow p_{+}$transition becomes faster than the $p_{+} \rightarrow s$ one at sufficiently strong fields [Fig. 4(c)], justifies the observation that electronic correlations at high magnetic field yield slightly increased relaxation rates. ${ }^{25}$ Yet, it may be worth

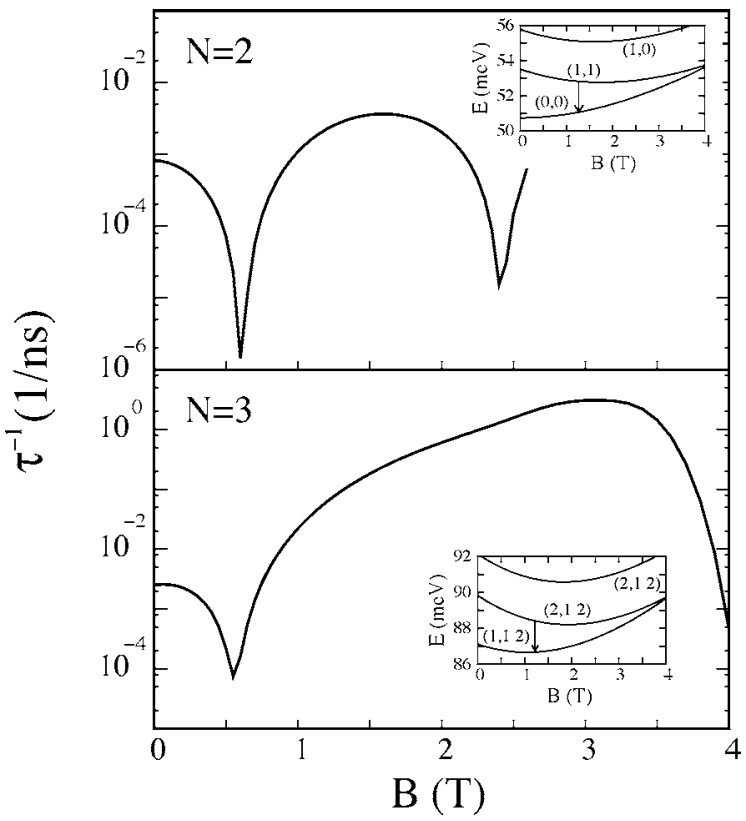

FIG. 5. Charge relaxation rate vs magnetic field for $N=2$ and 3 in a QD with $L_{z}=15 \mathrm{~nm}$ and $\hbar \omega_{0}=5 \mathrm{meV}$. Insets: lowest-lying energy levels along with their quantum numbers $(M, S)$; arrows indicate the transition under study.

pointing out that this takes place when the states involved in the transition have almost converged in energy (see inset in Fig. 3). Indeed, at such magnetic field values the investigated transitions are not the fundamental transition anymore, since higher-angular-momenta states have already come down in energy [this occurs at about $B=4 \mathrm{~T}$ in the QD we study, when the $(M=2, S=1)$ level becomes the ground state].

Recently, it has been suggested that electron-phonon coupling can be tailored in weakly-confined QDs and CQDs, so that SE lifetimes may be increased by orders of magnitude. ${ }^{7,11-13}$ The physical idea behind this possibility is to achieve an antiphase relation between the phonon wave and the electron wave function along the growth direction of the QD structure, i.e., to make the phonon wavelength along $z$ a divisor of the quantum well width, thereby reducing strongly the value of the form factor in Eq. (3). The phonon wavelength can be controlled through the lateral confinement, which is in turn determined either by electrostatic fields or by an axial magnetic field. The latter reduces the energy splitting between the low-lying SE states and thus increases the phonon wavelength in a controllable manner. ${ }^{7,12,13}$ The possibility of using external fields to suppress charge relaxation also in ME systems may pose a significant support for the eventual fabrication of ME QD devices with small decoherece rates. However, the applicability is not straightforward: indeed, as mentioned before, the energy of the emitted phonon corresponding to the fundamental transition decreases with increasing $N$, owing to the larger density of states. ${ }^{26}$ As a result, the phonon wavelength may be too long to ever match antiphase relation with the electron wave function in the growth direction. In order to explore this issue, in Fig. 5 we represent the charge relaxation rate in a QD with $\hbar \omega_{0}=5 \mathrm{meV}$ and $L_{z}=15 \mathrm{~nm}$ with $N=2$ and 3 


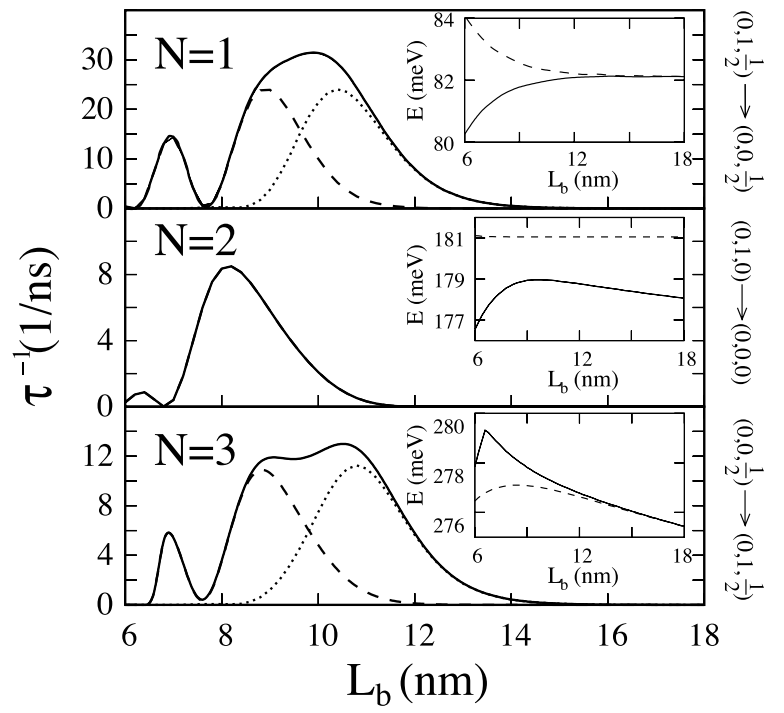

FIG. 6. Charge relaxation rate vs interdot barrier thickness $L_{b}$ in a CQD with $L_{z}=5 \mathrm{~nm}, \hbar \omega_{0}=5 \mathrm{meV}$, and $N=1,2,3$ electrons. Solid lines: total scattering rate. Dashed lines: DP contribution. Dotted lines: PZ contribution. Note the different vertical scale in each panel. Next to the right axis, we indicate the quantum numbers $(M, G, S)$ of the states involved in the transition. For $N=2$, the PZ contribution cannot be distinguished on this scale. Insets: lowestlying symmetric (solid line) and antisymmetric (dashed line) energy levels.

electrons. For $N=2$, two scattering minima show up at $B$ $=0.6$ and $2.4 \mathrm{~T}$. These are the same values as found in the $N=1$ case $^{7}$ which suggests that the $N=2$ scattering is well described as an independent-particle event. However, the $N$ $=3$ picture only shows one minimum at $B=0.55 \mathrm{~T}$ [the dip at $B \sim 4 \mathrm{~T}$ is simply due to the crossing between the $(M=1, S$ $=1 / 2)$ and $(M=2, S=1 / 2)$ energy levels]. This is a clear manifestation of phonon energy reduction due to Coulomb interactions. For a larger number of electrons (not shown), when the density of states is still higher, the magnetic field brings about frequent changes of energy levels with different symmetry, so that tuning the emitted phonon energy is no longer feasible. We then conclude that $B$-induced suppression of charge relaxation rates holds for QDs with a small enough number of electrons, but it rapidly loses efficiency as the number of particles increases, owing to the increasing density of states.

\section{COUPLED QUANTUM DOTS}

In this section we study charge relaxation rates corresponding to $N$-electron isospin transitions in vertically CQDs as a function of the interdot barrier thickness $L_{b}$. The two QDs are supposed to be identical. ${ }^{27}$ We focus on the transition between the lowest symmetric $(G=0)$ and antisymmetric $(G=1)$ solutions of the double quantum well with the spin quantum numbers of the ground state, i.e., the fundamental isospin (interdot) transition. This is indeed the fundamental transition of the system when the tunneling energy is smaller than the lateral confinement energy of the constituent QDs.

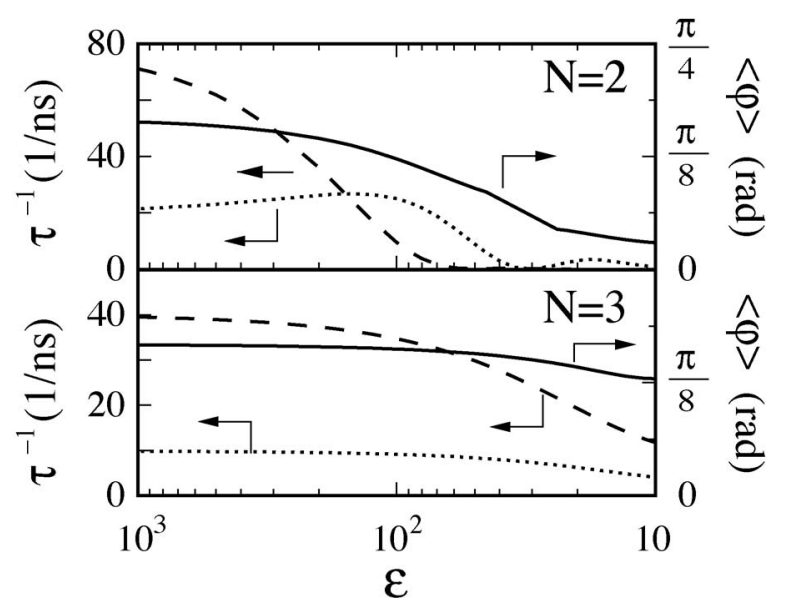

FIG. 7. Charge relaxation rate (left axis) and mean angle of phonon emission (right axis) vs effective dielectric constant $\varepsilon$ in the CQD structure of Fig. 6 at $L_{b}=10 \mathrm{~nm}$, for $N=2,3$. Solid lines: mean emission angle $(\varphi=0$ corresponds to emission along the growth direction). Dashed lines: DP scattering rate. Dotted lines: PZ scattering rate.

Figure 6 illustrates the results for a CQD structure with $L_{z}=5 \mathrm{~nm}, \hbar \omega_{0}=5 \mathrm{meV}$, and $N=1,2,3$ electrons. Solid lines indicate the total scattering rate, while dashed and dotted lines represent the contribution coming separately from DP and PZ interactions, respectively. As for the intradot transition case, it is worth noting that the $N=1$ calculation represents the independent-particle limit of the $N=2$ and 3 systems: one electron relaxes from the $\chi_{1}$ to the $\chi_{0}$ orbital while the other electrons remain static. Since the maximum scattering rate of the $N=2(N=3)$ system is smaller than that of $N=1$ by a factor of about 4 (2), we conclude that interdot transitions also benefit from a correlation-induced reduction of the scattering rates. Such reduction can be interpreted in terms of configuration mixing in analogous manner to the intradot case described in the previous section.

We note in Fig. 6 that the shape of the scattering rate curve is qualitatively similar for $N=1$ and 3 , with a dominating and oscillating DP scattering at small barrier thickness, and a PZ contribution which becomes dominant as the barrier thickness exceeds $\sim 10 \mathrm{~nm} .^{7}$ In contrast, the shape significantly differs for $N=2$, where the PZ contribution is missing. Actually, a close inspection reveals that it has been suppressed by almost four orders of magnitude.

The origin of the PZ scattering suppression can be elucidated from Fig. 7, corresponding to the CQD at $L_{b}=10 \mathrm{~nm}$, where we compare the DP and PZ scattering rate (dashed and dotted lines, respectively) and the mean angle of phonon emission $\langle\varphi\rangle$ (solid line) for $N=2$ and 3 , as a function of the Coulomb interaction strength. Here, $\varphi=0$ corresponds to emission along the growth direction, and the strength of the Coulomb interaction is simulated by changing the effective dielectric constant, which is, however, fixed at $\varepsilon=12.9$ in Eqs. (5) and (6) because we are interested in modulating electron-electron interaction only. The figure reveals a clear correlation between the Coulomb interaction strength and the emission angle of the phonon. In both cases the stronger the 
interaction the smaller the angle, but this effect is much more pronounced in the $N=2$ case. Since the PZ contribution is proportional to the phonon momentum projections [see Eqs. (5) and (6)], for the very small angles of $N=2$ at realistic values of the dielectric constant, the reduced in-plane components of the phonon momentum $\left(q_{x}, q_{y}\right)$ make PZ interaction neglegible. On the other side, the DP contribution is not so affected by the emission angle since it only depends on the modulus of the phonon momentum [Eq. (4)]. The characteristic angular dependence of the $N=2$ system follows from a complex interplay between its ME states configuration and the phonon orientation in isospin transitions of CQDs. The latter factor is a SE feature and will be studied elsewhere. We conclude this section stressing that the suppression of PZ scattering is a robust phenomenon for twoelectron interdot transitions and usual values of semiconductor dielectric constants. This may constitute an advantage of $N=2$ systems as compared to $N=1$ ones.

\section{SUMMARY}

We have investigated the effect of Coulomb interaction on the charge relaxation rates of disk-shaped QD systems with few electrons, due to incoherent coupling with acoustic phonons. We have studied both intradot transitions in single
QDs and interdot (isospin) transitions in vertically CQDs. Coulomb interaction affects the scattering rates in two ways: first, it changes the emitted phonon energy and momentum, and second, it changes the electron wave function through electronic correlations. Both effects have a significant influence on the electron relaxation rate in a way that generally leads to a reduction of the intradot and interdot transition rates. This trend is gradually quenched by axial magnetic fields. On the other hand, the increasing density of states with higher number of particles reduces the energy of the emitted phonons, which renders PZ interaction increasingly important, so that it may eventually become the dominant scattering mechanism even at zero magnetic field. We have finally shown that the suppression of electron scattering in weakly-confined QDs, which was recently suggested for SE systems, ${ }^{7,12,13}$ also applies to multielectron QDs with a sufficiently small number of particles.

\section{ACKNOWLEDGMENTS}

We acknowledge support from the Italian Ministry for University and Scientific Research under FIRB RBIN04EY74, CINECA Calcolo parallelo 2006, EU under the TMR network "Exciting" and Marie Curie IEF project MEIF-CT-2006-023797.
*Electronic address: climente@unimo.it; URL: http:// www.nanoscience.unimo.it/

${ }^{1}$ T. Fujisawa, D. G. Austing, Y. Tokura, Y. Hirayama, and S. Tarucha, Nature (London) 419, 278 (2002).

${ }^{2}$ T. Fujisawa, D. G. Austing, Y. Tokura, Y. Hirayama, and S. Tarucha, J. Phys.: Condens. Matter 15, R1395 (2003).

${ }^{3}$ T. Fujisawa, T. H. Oosterkamp, W. G. van der Wiel, B. W. Broer, R. Aguado, S. Tarucha, and L. P. Kouwenhoven, Science 282, 932 (1998).

${ }^{4}$ S. Tarucha, T. Fujisawa, K. Ono, D. G. Austing, T. H. Oosterkamp, W. G. van der Wiel, and L. P. Kouwenhoven, Microelectron. Eng. 47, 101 (1999).

${ }^{5}$ G. Ortner, R. Oulton, H. Kurtze, M. Schwab, D. R. Yakovlev, M. Bayer, S. Fafard, Z. Wasilewski, and P. Hawrylak, Phys. Rev. B 72, 165353 (2005).

${ }^{6}$ U. Bockelmann, Phys. Rev. B 50, 17271 (1994).

${ }^{7}$ J. I. Climente, A. Bertoni, G. Goldoni, and E. Molinari, Phys. Rev. B 74, 035313 (2006).

${ }^{8}$ V. N. Stavrou and X. Hu, Phys. Rev. B 72, 075362 (2005).

${ }^{9}$ Z. J. Wu, K. D. Zhu, X. Z. Yuan, Y. W. Jiang, and H. Zheng, Phys. Rev. B 71, 205323 (2005).

${ }^{10}$ P. Stano and J. Fabian, cond-mat/0604633 (to be published).

${ }^{11}$ P. Zanardi and F. Rossi, Phys. Rev. Lett. 81, 4752 (1998); P. Zanardi and F. Rossi, Phys. Rev. B 59, 8170 (1999).

${ }^{12}$ A. Bertoni, M. Rontani, G. Goldoni, F. Troiani, and E. Molinari, Appl. Phys. Lett. 85, 4729 (2004).

${ }^{13}$ A. Bertoni, M. Rontani, G. Goldoni, F. Troiani, and E. Molinari, Physica E (Amsterdam) 26, 427 (2005).

${ }^{14}$ S. Sasaki, T. Fujisawa, T. Hayashi, and Y. Hirayama, Phys. Rev. Lett. 95, 056803 (2005).
${ }^{15}$ J. M. Taylor, J. R. Petta, A. C. Johnson, A. Yacoby, C. M. Marcus, and M. D. Lukin, cond-mat/0602570 (to be published) and references therein.

${ }^{16}$ M. Brasken, S. Corni, M. Lindberg, J. Olsen, and D. Sundholm, Mol. Phys. 100, 911 (2002).

${ }^{17}$ A. Bertoni, M. Rontani, G. Goldoni, and E. Molinari, Phys. Rev. Lett. 95, 066806 (2005).

${ }^{18}$ S. Tarucha, D. G. Austing, S. Sasaki, Y. Tokura, W. van der Wiel, and L. P. Kouwenhoven, Appl. Phys. A: Mater. Sci. Process. 71, 367 (2000); U. Bockelmann, Ph. Roussignol, A. Filoramo, W. Heller, G. Abstreiter, K. Brunner, G. Böhm, and G. Weimann, Phys. Rev. Lett. 76, 3622 (1996); J. Kyriakidis, M. PioroLadriere, M. Ciorga, A. S. Sachrajda, and P. Hawrylak, Phys. Rev. B 66, 035320 (2002).

${ }^{19}$ S. M. Reimann and M. Manninen, Rev. Mod. Phys. 74, 1283 (2002).

${ }^{20}$ M. Rontani, C. Cavazzoni, D. Bellucci, and G. Goldoni, J. Chem. Phys. 124, 124102 (2006).

${ }^{21}$ Physics of Hot Electron Transport in Semiconductors, edited by C. S. Ting (World Scientific, Singapore, 1992).

${ }^{22}$ Group IV Elements and II-V Compounds, edited by O. Madelung, Landolt-Börnstein, Vol. 17 Semiconductors (Springer-Verlag, Berlin, 1982).

${ }^{23}$ The suppression of the relaxation rate at small confinement energies for $N=1$, is not seen in Fig. 1 because it occurs for characteristic frequencies $\hbar \omega_{0}<0.1 \mathrm{meV}$, but it is apparent, e.g., in Fig. 2(c).

${ }^{24}$ The large shift from $N=2$ to $N=3$ is due to the occupation of the $p$-shell by the ground state electrons, which greatly reduces the kinetic energy difference with respect to the excited states. 
${ }^{25}$ It may be worth pointing out that the phonon energy of the plotted transitions in Fig. 4 is identical, so the different rates are due to orbital effects only.

${ }^{26}$ Note that the role of electronic correlations is secondary for this discussion since we are concerned about the electron wave func- tion in the vertical direction only, which is the first solution of the quantum well for all low-lying QD states due to the strong vertical confinement.

${ }^{27}$ The scattering rates of SE CQDs with slightly anisotropic QDs have been studied in Ref. 7 . 